\title{
Documentation in a Medical Setting: Effects of Technology on Perceived Quality of Care
}

\author{
Julia DeBlasio and Bruce N. Walker \\ Sonification Lab, Georgia Institute of Technology \\ Atlanta, Georgia, USA 30332-0170 \\ julia@gatech.edu,bruce.walker@psych.gatech.edu
}

\begin{abstract}
The authors examine the social impact of introducing advanced exam-room technologies to the doctorpatient interaction. A total 342 participants viewed one of several video conditions portraying a physician conducting a medical interview in which he used one of 5 documenting methods/devices (nothing, pen and paper, PDA, desktop computer, wearable computer). After viewing the interaction, participants completed a series of questionnaires evaluating their general satisfaction with the quality of care (QoC) delivered during the medical interview. Results reveal that the type of technology used has a significant effect on QoC ratings. Though advanced technology offers the opportunity of better healthcare delivery, there may be a trade-off with lower ratings of interpersonal interactions.
\end{abstract}

\section{INTRODUCTION}

The quality of care (QoC) perceived by a patient during the medical encounter has implications for patient recovery and health maintenance. Patients who believe they have received poor QoC are less likely to adhere to doctor recommendations. A major influence on perceived QoC is the communication, both verbal and non-verbal, between the doctor and patient. The medical interview is the portion of the interaction when the doctor assesses the patient's symptoms and concerns. During the medical interview, a doctor must take note of the patient's comments while continuing to listen and prompt the patient with more probing questions in order to elicit all potentially relevant health related information.

Physicians have several options as to which note-taking medium to utilize. Traditionally, pen and paper have been used to jot down notes during the course of the examination. Alternatively, doctors may have chosen to make mental notes during the examination and to document these notes after exam completion. Advanced technology can potentially be utilized as a powerful tool in the exam room; allowing the physician to seamlessly access patient history, up-to-date medical information, and possible diagnoses - all while conversing with the patient.

Technology use in the exam room is not, however, without drawbacks. The presence of a device meant to enhance the medical encounter will necessarily alter the nature of both verbal and non-verbal components of the interaction. Understanding how patients will react (through measurable changes in perceived QoC) to exam room technology and, more specifically, to resultant changes in the doctor-patient interaction is key in understanding how to counter any negative consequences. The benefits of assistive technologies cannot fully be realized until patient barriers to technology adoption are addressed.

\section{Doctor-Patient Interactions}

Arguably, the interaction between doctor and patient during the medical consultation is the most critical point for transferring information and the delivery of excellent healthcare (Bertakis, Roter, \& Putnam, 1991; Ong, de Haes,
Hoos, \& Lammes, 1995; Russuvuori, 2001). The physician's primary task is to become familiar with patient history while eliciting symptoms in a way that is meaningful. A secondary, but nearly as important, task is for the physician to connect with the patient. The doctor-patient interaction is the patient's most salient feature on which to judge the QoC they receive.

There are at least two aspects to healthcare quality: actual patient outcome (observable consequences due to a medical encounter); and perceived QoC (the patient's personal judgment of the healthcare quality). Actual patient outcome can be measured in several ways including: adherence to doctor recommendations; recall of information given during consultation; and understanding of diagnosis (Ong et al., 1995). Perceived QoC is a good predictor of actual patient outcome (Ong et al.). The most widely accepted assessment of perceived QoC, and the measure that is considered in this study, is patient satisfaction. Patient satisfaction will be operationalized in subsequent sections. Ong et al. report that patients evaluate their overall healthcare experience on their doctor's interpersonal skills; skills which are interpreted through both verbal and (largely) non-verbal communication.

\section{Verbal Versus Non-verbal Communication}

Verbal communication consists not only of the spoken word, but also of verbal inflection, pauses in speech, and tone. Nonverbal communication has been operationalized as body positioning, posture, gaze, etc. These non-verbal components, or visual cues, make up approximately $80 \%$ of perceived interpersonal communication (Ong et al., 1995). The introduction of exam room technology will change both verbal and non-verbal aspects of communication by constraining the office layout and acting as a third entity in an otherwise dyadic interaction.

Previous research has shown that patients often believe that their physician is not listening to them when attention is shifted from the patient to the records; resulting in loss of eye contact (Ruusuvuori, 2001). For an anxious patient, the need to know the physician is engaged in the conversation is heightened. Commonly, tactics are employed by speakers to regain eye contact with an intended recipient whose gaze has wandered. One such tactic is achieved by pausing mid- 
sentence, or engaging in other speech discontinuities until the recipient's gaze is regained (Goodwin, 1981). This verbal tactic has been observed during medical interviews, indicating that the patient is perturbed by the loss of their physician's gaze (Ruusuvuori).

\section{Exam Room Note Taking}

Note taking affords a crucial written record without which the doctor might tend to overlook problems presented subsequent to the beginning of the medical interview (Ruusuvuori, 2001). The method used by the doctor to take notes may affect the layout of the exam room and the physician's physical posture. Physical posture constrains both eye contact and body orientation of the doctor to the physician (Gorawara-Bhata, Cook, \& Sachsa, 2006).

Eye contact is broken when the physician shifts attention from the patient to refer to medical records or to input notes. Even when eye contact is maintained, the speaker's torso may or may not be facing the recipient depending on the constraints of the workspace. The physician's torso may be squarely facing the patient $\left(0^{\circ}\right.$ body orientation) or the physician's torso may be oriented away from the patient $\left(90^{\circ}\right.$ body orientation). In the second case, the physician must turn his head to face the patient (see Figure 1). Evidence has shown that people prefer the $0^{\circ}$ body orientation to the $90^{\circ}$ when speaking to someone (Ruusuvuori, 2001; Furnham, Petrides, \& Temple, 2006; Ong et al., 1995). A recent emphasis on patient-centered medical care has created a need for questions about patient preferences to be answered. Despite a shift in the medical field towards more patientcentered solutions, few studies have addressed the social impact of implementing advanced technology in the medical interview. Thus, we compared reactions to multiple exam room technologies along the lines of patient satisfaction.

\section{METHOD}

\section{Participants}

Experiment 1. One hundred forty-two undergraduate students (78 male, 64 female) participated in exchange for extra credit in a psychology course. Participants ranged from 18 to 25 years with a mean age of 19.9 years.

Experiment 2. Two hundred different undergraduate students (103 male, 97 female) participated for extra credit, and ranged from 18 to 25 years with a mean age of 19.6 years.

\section{Stimuli}

Videos. In this between-subjects design, each participant watched one of ten brief videos depicting a doctor conducting a medical interview with his patient. During the interview, the doctor is ascertaining the patient's symptoms and concerns. The "doctor" in the video is a young, Caucasian male wearing a white lab coat and stethoscope (see Figure 1). The video was filmed from the point of view of the patient, who is sitting approximately three feet in front of the physician. The voice of a male patient can be heard on the video while he reports symptoms of an upper respiratory infection or a common cold, such as: headache, fatigue, loss of appetite, and coughing. Participants can see, as well as hear, the doctor. The film is set

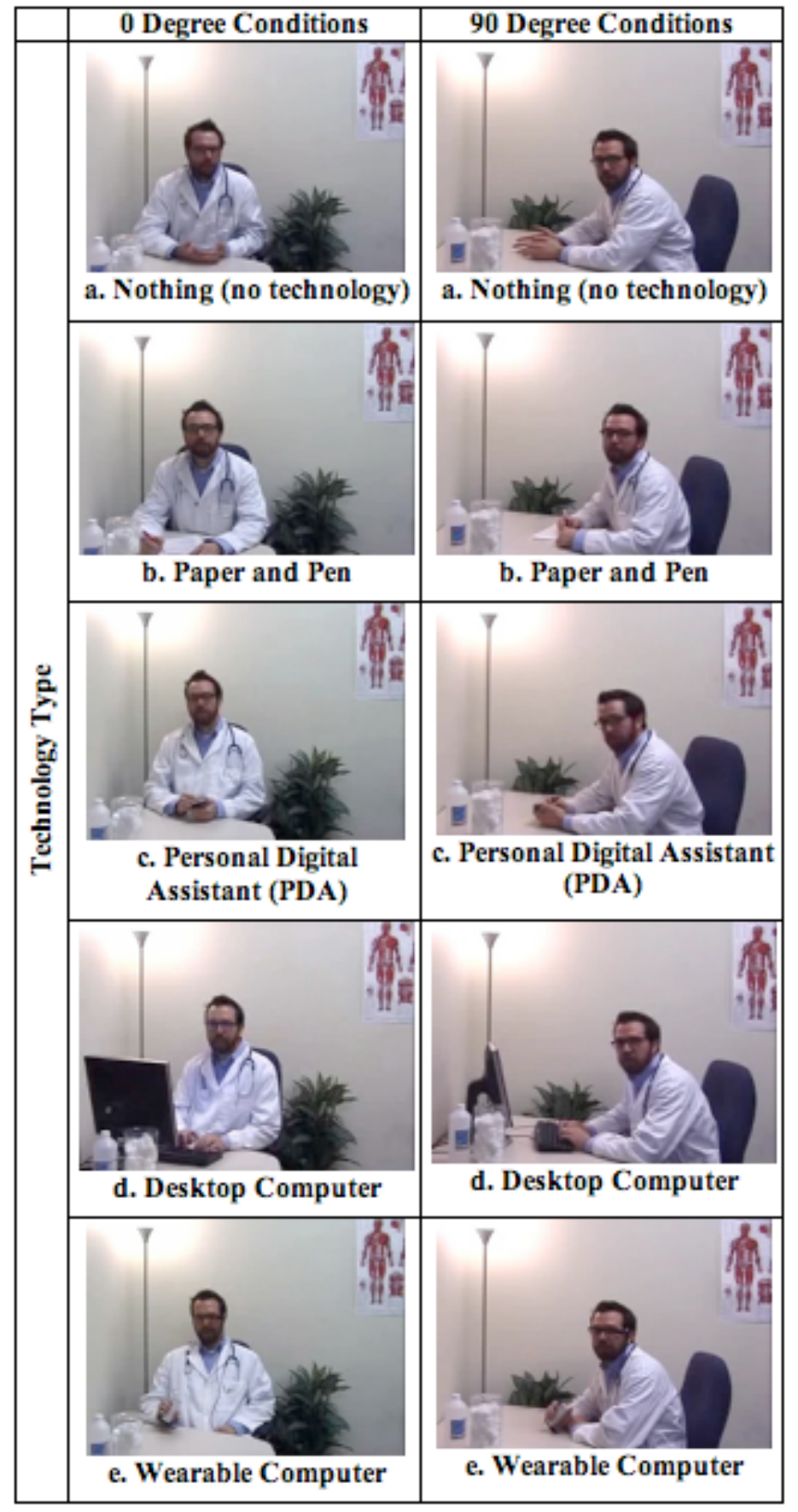

Figure 1. Still clips from each of the video conditions.

in a mock-up of a doctor's office including a desk, lamp, medical poster, jar of cotton balls, and plant. The scenario in the video reflects an ordinary, non-emotional visit to the doctor's office that any undergraduate would experience for a common illness. To control the dialogue across all videos, the patient's verbal responses were recorded in advance and this was overlaid onto the audio track for all videos.

Viewing. The DVD-quality videos were played from an LG DN6788 DVD player, and projected from a Dell 3200MP LCD projector onto a standard projection screen at a viewing distance of approximately 10 feet. The audio was played at comfortable listening levels through Altec Lansing 3-piece computer speakers. Between one and eight participants per 
session sat facing the screen to watch the video; however, participants' subsequent responses were completed individually. The projected size of the video was approximately 5 x 6.5 feet (see Figure 1).

Questionnaires. Four questionnaires were used in this study to assess each participant's satisfaction with the apparent QoC demonstrated in the video. These included a Background Questionnaire, a Quality of Care survey, an After Video Response sheet, and a Technology Use survey. The Background Questionnaire was used to collect demographic information as well as answers to questions such as, "Are you in good health?" The Quality of Care survey consists of 25 questions that address five subscales of QoC: Technical Quality (TECH), General Satisfaction (GSAT), Interpersonal Aspects (INTER), Communication (COMM), and Time Spent with the Doctor (TIME). The After Video Response sheet gave participants a chance to respond freely whether they would choose to go to this doctor, comment about the video, or comment about the study in general. The Technology Use survey established the participant's familiarity and regular use of a variety of technologies such as a cellular phone or cruise control (Czaja et al., 2001).

QoC Subscale Details. Questions under the subscale TECH addressed the doctor's technical competence. For instance, did the doctor seem to know what he was doing? GSAT subscale questions dealt with the participant's overall approval of the interaction they viewed. Questions within the INTER subscale dealt with the doctor's interpersonal skills. For instance, did he treat the patient with respect? Did the doctor make eye contact with the patient? COMM subscale questions assessed whether participants believed the doctor communicated clearly with the patient. Was the patient able to understand what the doctor said? Finally, the TIME subscale questions addressed whether the doctor spent adequate time with the patient.

\section{Variables}

Independent variables. The independent variables included the type of technology used by the physician to input the patient's responses and the orientation of the physician relative to the patient. Five technology conditions were used (nothing, pen and paper, PDA, desktop computer, and wearable computer) along with two physician-patient orientations ( 0 degrees and 90 degrees) for a total of ten conditions, each represented in videos of length 2 minutes and 34 seconds \pm 7 seconds (see Figure 1). The 0 degree condition is the case when the doctor is sitting while directly facing the patient. The 90-degree condition is the case when the doctor is facing approximately 90 degrees away from the patient so that he must torque his head to make direct eye contact (see Figure 1).

Dependent variables. The dependent variable in this study is participant general satisfaction and is operationally defined by participant responses to the After Video Questionnaire and the Quality of Care Survey. Specifically, the response to the question, "Would you go to this doctor" was used as a measure of participants' acceptance of the doctor's health care delivery. The 25 questions on the Quality of Care survey were categorized into five subscales: TECH, GSAT, INTER, COMM, and TIME.
Table 1. Brief Technology explanations given to Experiment 2 participants prior to viewing the video.

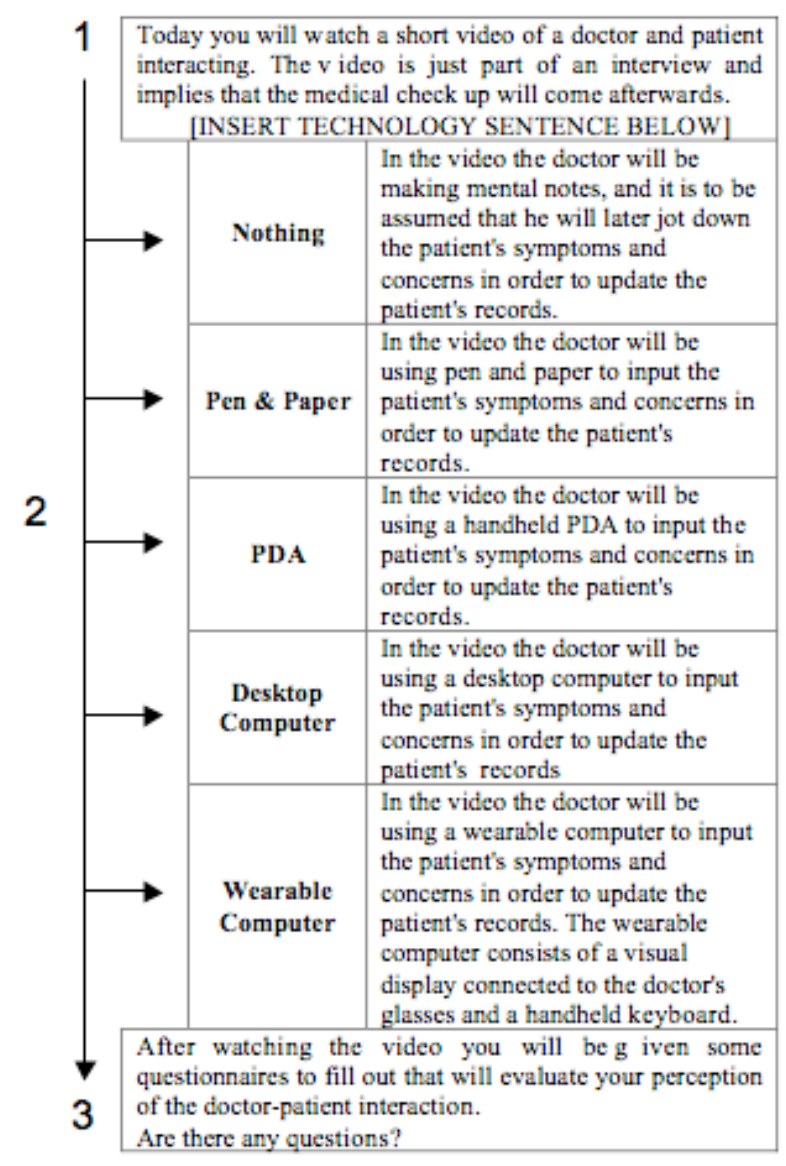

Other examined factors. In addition to the independent variables, the role of participant gender, academic major, age, recent health, and familiarity with technology were examined.

\section{Technologies}

The use of the word "technologies" in this study is used to mean the device or method used by the healthcare provider to take notes throughout the entire medical interview. These devices and methods are either currently used or could easily be implemented in a doctor's office (see Figure 1).

- Nothing: This condition represents the case where the physician does not document the medical interview, but rather relies on his own memory to update the patient's records.

- Paper and pen: The doctor takes hand-written notes onto a pad or directly onto the patient's chart.

- Personal Digital Assistant (PDA): The doctor is able to electronically store the patient's responses with a PDA and stylus. Additionally, the doctor is able to consult reference material such as patient history or drug interactions.

- Desktop Computer: This condition provides all the same functions as the PDA, but is visibly more noticeable. The doctor uses a keyboard and mouse as input devices to the desktop computer.

- Wearable Computer: The wearable computer consists of a small display attached to the physician's glasses (which may 


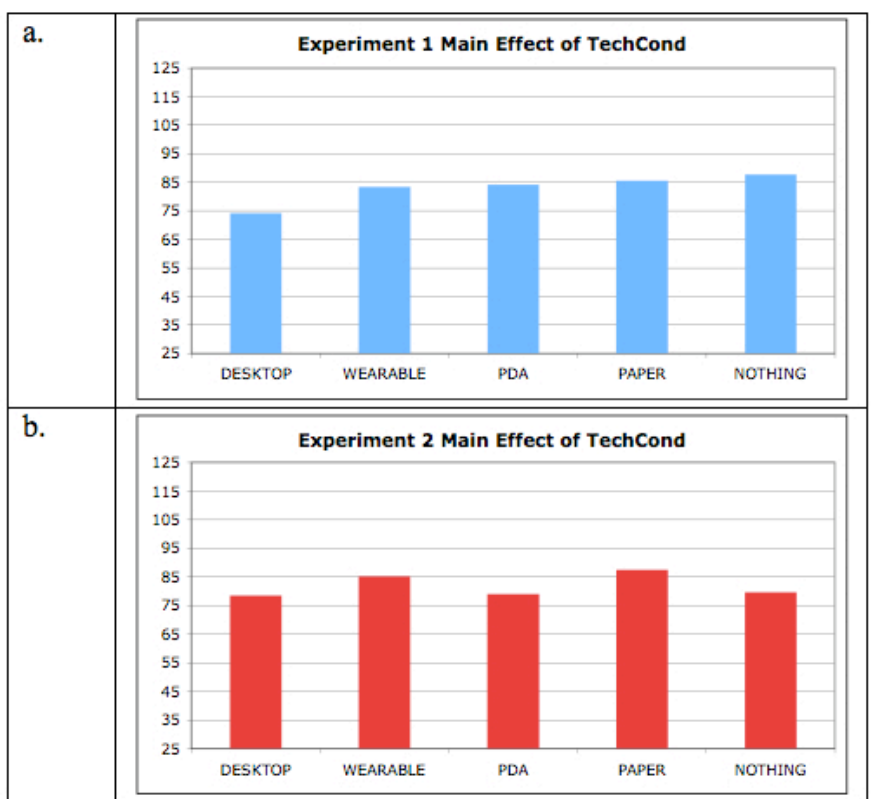

Figure 2. Effect of Tech Condition on QoC. Y-axis is AllQoC. (a) Exp. 1 Desktop is rated lower than all other technology conditions. (b) Exp. 2: Paper and Wearable are rated higher than the remaining technology conditions.

or may not be noticed) and a handheld keyboard known as a Twiddler, operated by the doctor using his right hand.

\section{Design and Procedure}

After completing the informed consent Background Questionnaire, participants were told that they would be viewing a brief video of a doctor and patient talking. Participants were also told that the interaction in the video was only part of the visit and to assume that the physical examination would follow the initial medical interview. At this point, the type of technology to be used in the participant's particular condition was brought to their attention for Experiment 2 participants only. See Table 1 for the scripted technology explanations. This step differs from Experiment 1, in which measures were taken to ensure the use of technology was not mentioned at all until the debriefing stage. Lastly, participants were informed that they would be given some questionnaires to complete after watching the video that would evaluate their perception of the doctorpatient interaction. Once the video ended, the participants completed the Quality of Care Questionnaire, After Video Response sheet, and Technology Use survey, in that order. Finally, the participants were debriefed.

\section{RESULTS}

Efforts were made to ensure the video conditions did not differ in any aspects except for the technological device or method used by the doctor to take notes during the medical interview. Therefore, any differences in the data collected from one video to the next were attributed to the technological device or method used, and not to some other difference between videos. Experiments 1 and 2 were analyzed independently, compared, and then combined for final analysis.

\section{Main Effect of Technology Condition}

First, the proportions of participants reporting that they would

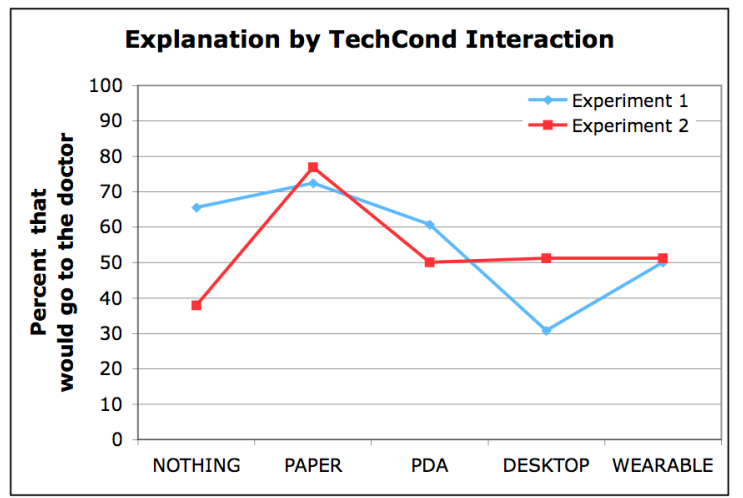

Figure 3. Average cumulative quality of care scores for each technology condition.

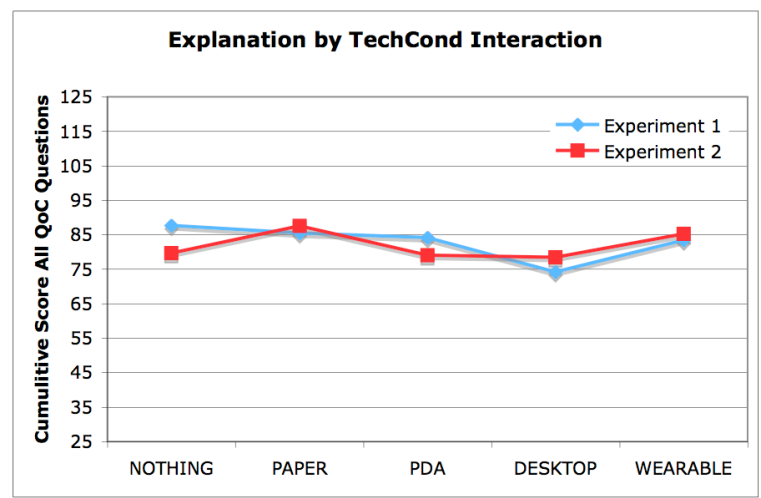

Figure 4. Percent of total participants in each technology group reporting that they would go to the depicted doctor if they had a medical problem.

go to the doctor (YesGo) were compared across all technology conditions. Univariate ANOVAs with YesGo as the dependent measure revealed a main effect of technology condition: for Experiment 1, $F(4,137)=3.063, p=0.019$; for Experiment 2, $F(4,193)=3.284, p=0.012$; and for combined results, $F(4,335)=4.104, p=0.003$.

An ANOVA using an aggregate score of ratings on all 25 QoC questions (AllQoC) as the dependent measure also revealed a main effect of technology condition. The desktop condition was rated significantly lower than all other technology conditions in Experiment 1 and both the nothing and wearable conditions were rated significantly higher than all other conditions in Experiment 2. These results are presented in Figure 2, which illustrates the rank order of all technology conditions for Experiment 1 and Experiment 2 separately using average score of AllQoC as a metric. Note that in Figures 2 and 4 the lowest aggregate score for all QoC questions ( 25 questions on a 1-5 scale) is 25 and the highest possible aggregate score is 125 . The analysis yielded the following results: $\mathrm{F}(4,137)=4.444, \mathrm{p}=0.002 ; \mathrm{F}(4,190)=$ $4.193, \mathrm{p}=0.003$; and $\mathrm{F}(4,327)=6.033, \mathrm{p}<0.001$ for Experiment 1, 2, and combined results, respectively.

A MANOVA using the five QoC subscales (GSAT, TECH, INTER,COMM, and TIME) as the dependent factors revealed a main effect of technology condition: $\mathrm{F}(4,137)=$ $2.162, p=0.003 ; \mathrm{F}(4,190)=2.479, p<0.001$; and $\mathrm{F}(4,332)=$ $3.129, p<0.001$ for Experiment 1, Experiment 2, and 
combined results, respectively.

\section{Technology Explanation Interaction}

The only procedural difference between the two experiments was the exclusion (Experiment 1) or the inclusion (Experiment 2) of a brief technology explanation. Therefore, statistical differences between experiments are attributed to the technology explanation. There were no main effects of technology explanation, however an ANOVA revealed a marginal interaction of explanation and technology condition (TechCond) with YesGo as the dependent measure F(4, 327) $=2.207, \mathrm{p}=0.068$. Figure 3 illustrates the difference in YesGo from Experiment 1 to Experiment 2 for each technology condition. A second ANOVA using AllQoC as the dependent measure shows an Explanation by TechCond interaction $\mathrm{F}(4$, $327)=2.865, p=0.023$ (see Figure 4). Further, a MANOVA using the QoC subscales revealed a marginal interaction $\mathrm{F}(4$, $327)=1.157, p=0.067$. A test of the between-subjects effects revealed that QoC subscales GSAT, INTER, and COMM show an Explanation by TechCond interaction with $\mathrm{F}(4,327)$ $=2.952, \mathrm{p}=0.020 ; \mathrm{F}(4,327)=2.770, \mathrm{p}=0.027$; and $\mathrm{F}(4,327)$ $=2.449, \mathrm{p}=0.046$, respectively. TIME and TECH subscales did not show an Explanation by TechCond interaction.

\section{DISCUSSION}

The fact that five of the five QoC subscales were significantly correlated at the $\mathrm{p}<0.001$ level with the YesGo variable (directly related to actual patient outcome) validates that responses on the QoC survey were a good measure of participants' perception of the quality of medical care delivered by the doctor. Further, the significant correlation between the majority of question pairs within each QoC subscale indicates that the questions within each subscale are measuring a common element.

Several dependent measures show that there is a main effect of technology condition. This main effect is compelling in light of the efforts made to make the videos as similar as possible in all aspects except for the technological device or method used by the doctor to take notes. Participants who were given no explanation of the technology (Experiment 1) rated the desktop computer lower on QoC questions than any other condition in either phase. Further, the desktop computer was the only condition in Experiment 1 with less than half of the participants reporting that they would go to the doctor depicted in the videos. Overall, without any explanation of purpose, the desktop computer is received the least favorably compared to all other conditions. Of course, this is a very important finding, given that doctors and health organizations continue to deploy desktop computers in many exam rooms.

Other technologies may be as useful as a desktop computer (or more so), and lead to greater patient satisfaction and QoC ratings. However, in such cases, giving the patients a brief explanation of the technological devices being used may lead to higher QoC ratings with a Desktop and Wearable computer. Curiously, ratings of the Nothing and PDA conditions decreased significantly with explanations. Future work is needed to elucidate patient reaction to specific notetaking devices and methods, but it may be that by pointing out that the doctor is not using technology to take notes may cause the patient to consider the doctor somewhat out of date, and possibly delivering lower-quality care. The clear conclusion here is that communicating with the patient so that they understand what a device is and why it is being used in the exam room is a good step to preventing a lowered perception of quality of care delivered.

By systematically evaluating changes in patient perceptions as technology is introduced into the medical exam room, we can ensure that both interpersonal communication and patient satisfaction are maximized, along with perceived and actual quality of medical care.

\section{REFERENCES}

Bertakis, K.D., Roter, D., \& Putnam, S.M. (1991). The relationship of physician medical interview style to patient satisfaction. Journal of Family Practice, 32(2), 175-177.

Czaja, S. J., Charness, N., Fisk, A. D., Rogers, W., \& Sharit, J. (2001). The center for research and education on aging and technology enhancement: A program for enhancing technology for older adults. Gerontology, 1, 50-59.

Czaja, S., Charness, N., Fisk, A. D., Hertzog, C., Nair, S., Rogers, W., et al. (2006). Factors predicting the use of technology: Findings from the center for Research and education on aging and technology enhancement (CREATE). Psychology and Aging, 21(2), 333-352.

DeBlasio, J., Walker, B., Caldwell, B., Mauney, L., Lyons, K., Kintz, E., et al. (2007). The use of different technologies during a medical interview: Effects on perceived quality of care (Tech. Rep. No. GIT-GVU-07-13). Atlanta, Georgia: The Georgia Institute of Technology, School of Psychology.

Goodwin, L., Prescott, P., Jacox, A., \& Collar, M. (1981). The nurse practitioner rating form: II. Methodological development. Nursing Research, Vol 30(5), 270-276.

Gorawara-Bhata, R., Cook, M. A., \& Sachsa, G. (2006). Nonverbal communication in doctor-elderly patient transactions (NDEPT): Development of a toolstar, open, star, openstar, open.

Ong, L.M., de Haes, J.C., Hoos, A.M., \& Lammes, F.B. (1995). Doctor-patient communication: A review of the literature. Social Science \& Medicine, 40(7), 903-918.

Robinson, T., White, G., \& Houchins, J. (2006). Improving communication with older patients: Tips from the literature. Family Practice Management website. Retrieved November 2007 from: www.aafp.org/fpm

Ruusuvuori, J. (2001). Looking means listening: Coordinating displays of engagement in doctor-patient interaction. Social Science \& Medicine. 52, 1093-1108.

Zeman, L., Johnson, D., Arfken, C., Smith, T., \& Opoku, P. (2006). Lessons learned: Challenges implementing a personal digital assistant (PDA) to assess behavioral health in primary care. Families, Systems, \& Health. 